\title{
Near-IR Fluorescent Molecular Hydrogen Emission from NGC 2023
}

\author{
Michael G. Burton ${ }^{1}$, J. E. Howe ${ }^{2}$, T. R. Geballe ${ }^{3}$ \\ and P. W. J. L. Brand ${ }^{4}$ \\ ${ }^{1}$ School of Physics, University of New South Wales, Sydney, NSW 2052, Australia \\ M.Burton@unsw.edu.au \\ ${ }^{2}$ Five College Radio Astronomy Observatory, Department of Physics and Astronomy, \\ University of Massachusetts, Amherst, MA 01003, USA \\ jhowe@lyra.phast.umass.edu \\ ${ }^{3}$ Joint Astronomy Centre, 660 N. A'ohoku Place, Hilo, HI 96720, USA \\ tom@jach.hawaii.edu \\ ${ }^{4}$ Institute for Astronomy, University of Edinburgh, Blackford Hill, \\ Edinburgh, EH9 3HJ, UK \\ pwb@roe.ac.uk \\ Received 1997 August 1, accepted 1998 January 20
}

\begin{abstract}
Spectra from 1 to $2.5 \mu \mathrm{m}$, at 230-430 spectral resolution, are presented of the fluorescent molecular hydrogen line emission from two locations in the reflection nebula NGC 2023. Over $100 \mathrm{H}_{2}$ lines can be identified in the spectra, although blending and poor atmospheric transmission mean that reliable level column densities can only be obtained from 35 lines. This latter group includes lines from $v=1-8$ and $v=10$, spanning an energy range from 6000 to $45,000 \mathrm{~K}$ above the ground state. These data may be used to constrain models of photodissociation regions and of fluorescent excitation for molecular hydrogen.
\end{abstract}

Keywords: molecular processes-ISM: molecules_reflection nebulae-ISM: individual sources (NGC 2023)

\section{Introduction}

The reflection nebula NGC 2023 is one of the brightest sources of fluorescent molecular hydrogen in the sky, making it a laboratory for the study of $\mathrm{H}_{2}$ fluorescence, which occurs over a wide range of physical conditions. It is powered by the $\mathrm{B} 1.5 \mathrm{~V}$ star HD 37903, the most luminous member of a cluster of young stellar objects illuminating the front surface of the Lynds 1630 molecular cloud in Orion B (Depoy et al. 1990). NGC 2023 forms a cavity in the surface of the cloud, some $450 \mathrm{pc}$ from us, producing both a bright visual reflection nebula and a UV-excited photodissociation region. The latter is evident through, for instance, extensive $\mathrm{C}^{+}$ $158 \mu \mathrm{m}$ line emission (Howe et al. 1991), extended red emission features (Witt \& Malin 1989) and IR emission features from PAHs (Joblin et al. 1995).

Fluorescent molecular hydrogen was first discovered through observations of NGC 2023 (Gatley et al. 1987; Hasegawa et al. 1987), on the basis of a $1-0 / 2-1 \mathrm{~S}(1)$ line ratio of $\sim 2$. Narrow line profiles
(FWHM $<16 \mathrm{kms}^{-1}$, Burton et al. 1990a), and numerous high-excitation emission lines emitted in the far red (from levels as high as $v=7 \& 8$, Burton et al. 1992) are also evidence for the fluorescent excitation process in this source, as opposed to shocks. Emission line images (Burton et al. 1989; Field et al. 1994) show narrow filamentary structure on top of a diffuse emission nebula, suggestive of emission from limb-brightened undulations in the surface of the molecular cloud. However, SteimanCameron et al. (1997) argue that elevated intensities are due, in large measure, to enhancements in local density rather than limb brightening.

The application of photodissociation region (PDR) models (e.g. Tielens \& Hollenbach 1985) to interpreting the emission in sub-mm CO rotational, far-IR fine structure and near-IR $\mathrm{H}_{2}$ lines from NGC 2023 (Jaffe et al. 1990; Burton, Hollenbach \& Tielens 1990b; Steiman-Cameron et al. 1997) suggests that a far-UV radiation field of $\sim 10^{4}$ times the average interstellar value is incident on a clumpy molecular cloud, with the bulk of the gas at densities of 


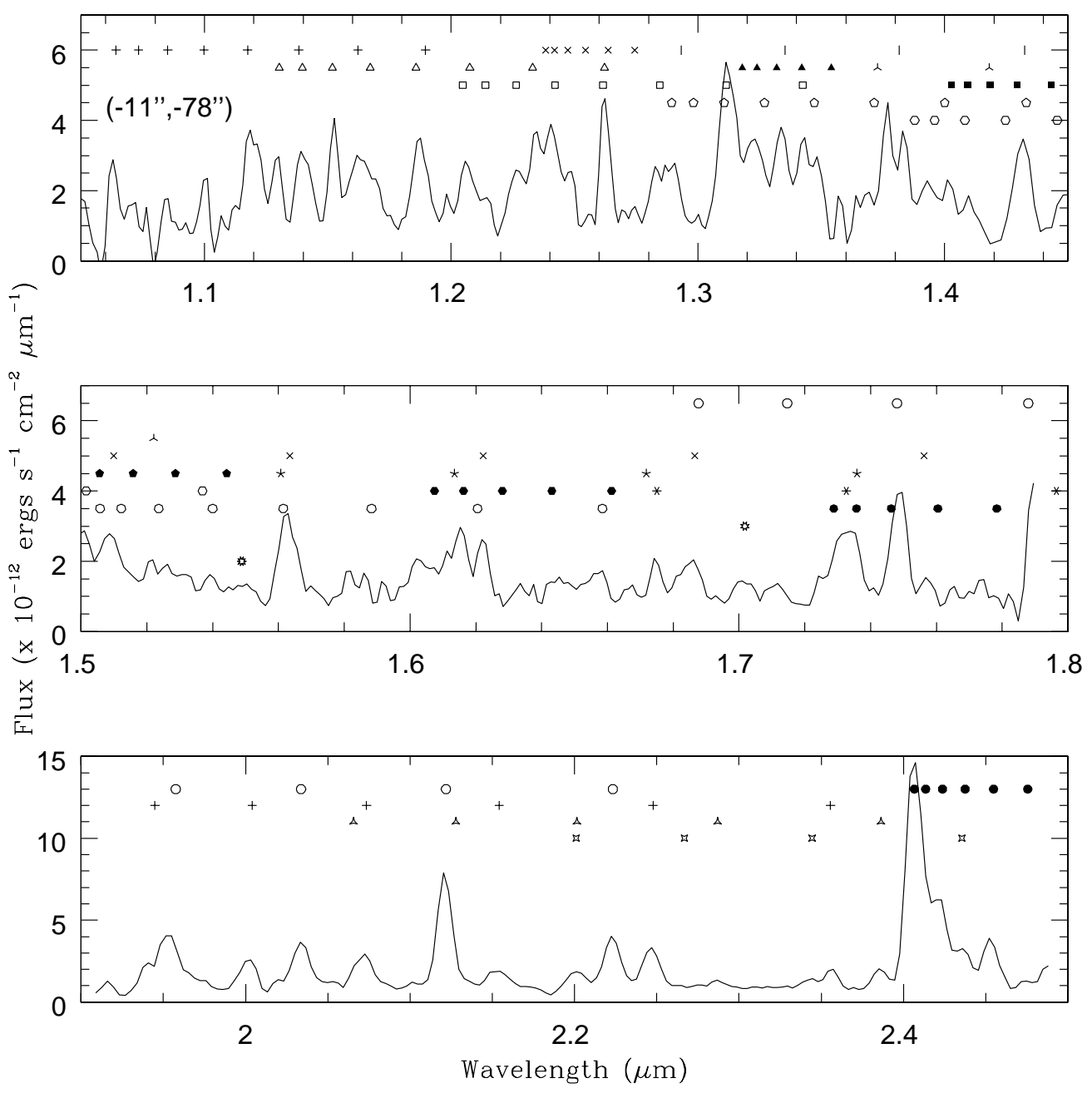

Figure 1-Spectra from $1-2 \cdot 5 \mu \mathrm{m}$ observed at the southern location $\left(-11^{\prime \prime},-78^{\prime \prime}\right)$ in NGC 2023 . Several series of vibrational-rotational lines of $\mathrm{H}_{2}$ are indicated. The wavelength of the lowest excitation line in each branch shown is described by a code as in the following example: $2-0 \mathrm{~S}(0)+, 1.190 \mu \mathrm{m}$ denotes the $2-0$ S-branch with + signs, starting from 2-0 S(0) at $1.190 \mu \mathrm{m}$. Top panel (J band): 2-0 $\mathrm{S}(0)+, 1.190 \mu \mathrm{m} ; 2-0 \mathrm{Q}(1) \times, 1.238 \mu \mathrm{m} ; 2-0 \mathrm{O}(2)$ vertical bar, $1.293 \mu \mathrm{m} ; 3-1 \mathrm{~S}(0)$ open triangle, $1.262 \mu \mathrm{m} ; 3-1 \mathrm{Q}(1)$ filled triangle, $1.314 \mu \mathrm{m} ; 3-1 \mathrm{O}(2)$ 3-sided star, $1.373 \mu \mathrm{m} ; 4-2 \mathrm{~S}(0)$ open square, $1.343 \mu \mathrm{m} ; 4-2 \mathrm{Q}(1)$ filled square, $1.398 \mu \mathrm{m} ; 5-3 \mathrm{~S}(0)$ pentagon, $1.433 \mu \mathrm{m} ; 6-4 \mathrm{~S}(3)$ hexagon, $1.446 \mu \mathrm{m}$. Middle panel (H-band): 1-0 S(6) open circle, $1.788 \mu \mathrm{m} ; 3-1 \mathrm{O}(5) 3$-sided star, $1.522 \mu \mathrm{m} ; 4-2 \mathrm{O}(3) \times, 1.510 \mu \mathrm{m} ; 5-3 \mathrm{Q}(3)$ filled pentagon, $1.506 \mu \mathrm{m} ; 5-3 \mathrm{O}(2)$ 5-sided star, $1.561 \mu \mathrm{m} ; 6-4 \mathrm{~S}(0)$ open hexagon, $1.537 \mu \mathrm{m} ; 6-4 \mathrm{Q}(1)$ filled hexagon, $1.602 \mu \mathrm{m} ; 6-4 \mathrm{O}(2) 6$-sided star, $1.675 \mu \mathrm{m} ; 7-5 \mathrm{~S}(0)$ open 7-gon, 1.658 $\mu \mathrm{m} ; 7-5 \mathrm{Q}(1)$ filled 7-gon, $1.729 \mu \mathrm{m} ; 8-6 \mathrm{~S}(3)$ 8-star, $1.702 \mu \mathrm{m} ; 10-7 \mathrm{~S}(3)$ 10-star, $1.549 \mu \mathrm{m}$. Bottom panel (K-band): 1-0 S(0) open circle, $2 \cdot 223 \mu \mathrm{m} ; 1-0 \mathrm{Q}(1)$ filled circle, $2 \cdot 407 \mu \mathrm{m} ; 2-1 \mathrm{~S}(0)+, 2 \cdot 356 \mu \mathrm{m} ; 3-2 \mathrm{~S}(1) 3$-gon, $2 \cdot 386 \mu \mathrm{m} ; 4-3 \mathrm{~S}(2)$ 4-star, $2 \cdot 435 \mu \mathrm{m}$.

$\sim 10^{3} \mathrm{~cm}^{-3}$, but with a fraction $(\sim 10 \%)$ about two orders of magnitude denser. However, we note that Wyrowski et al. (1997) suggested on the basis of C91 $\alpha$ radio data, that the beam filling factor for high-density gas is much greater than this, albeit with the dense clumps only at $100-300 \mathrm{~K}$ as opposed to the $750 \mathrm{~K}$ determined by Steiman-Cameron et al.

In view of its brightness, the $\mathrm{H}_{2}$ emission from NGC 2023 can be subjected to detailed spectral examination, with emission lines from highly-excited vibrational-rotational levels in the ground electronic state able to be measured. Such observations permit stringent tests of fluorescent excitation models (e.g. Black \& van Dishoeck 1987; Sternberg \& Dalgarno 1989; Burton, Hollenbach \& Tielens 1990b; Draine \& Bertoldi 1996) for $\mathrm{H}_{2}$. Deviations from a pure fluorescent cascade might result from collisional depopulation of excited levels, from formation pumping of newly created molecules, from variations in the injection levels for the cascade, and/or from changes in the ortho-para ratio. Thus they may be used to test our physical models for these processes. This paper reports observations of the $1-2 \cdot 5 \mu \mathrm{m} \mathrm{H}_{2}$ emission from two locations in NGC 2023 which can be used for this purpose. Parts of these data have previously been discussed in the $\mathrm{PhD}$ Dissertation of John Howe (1992) and in a review on molecular hydrogen emission (Burton 1992).

\section{Observations}

The spectra presented here were obtained on UT 1989 November 28-December 1 and 1991 January 19 

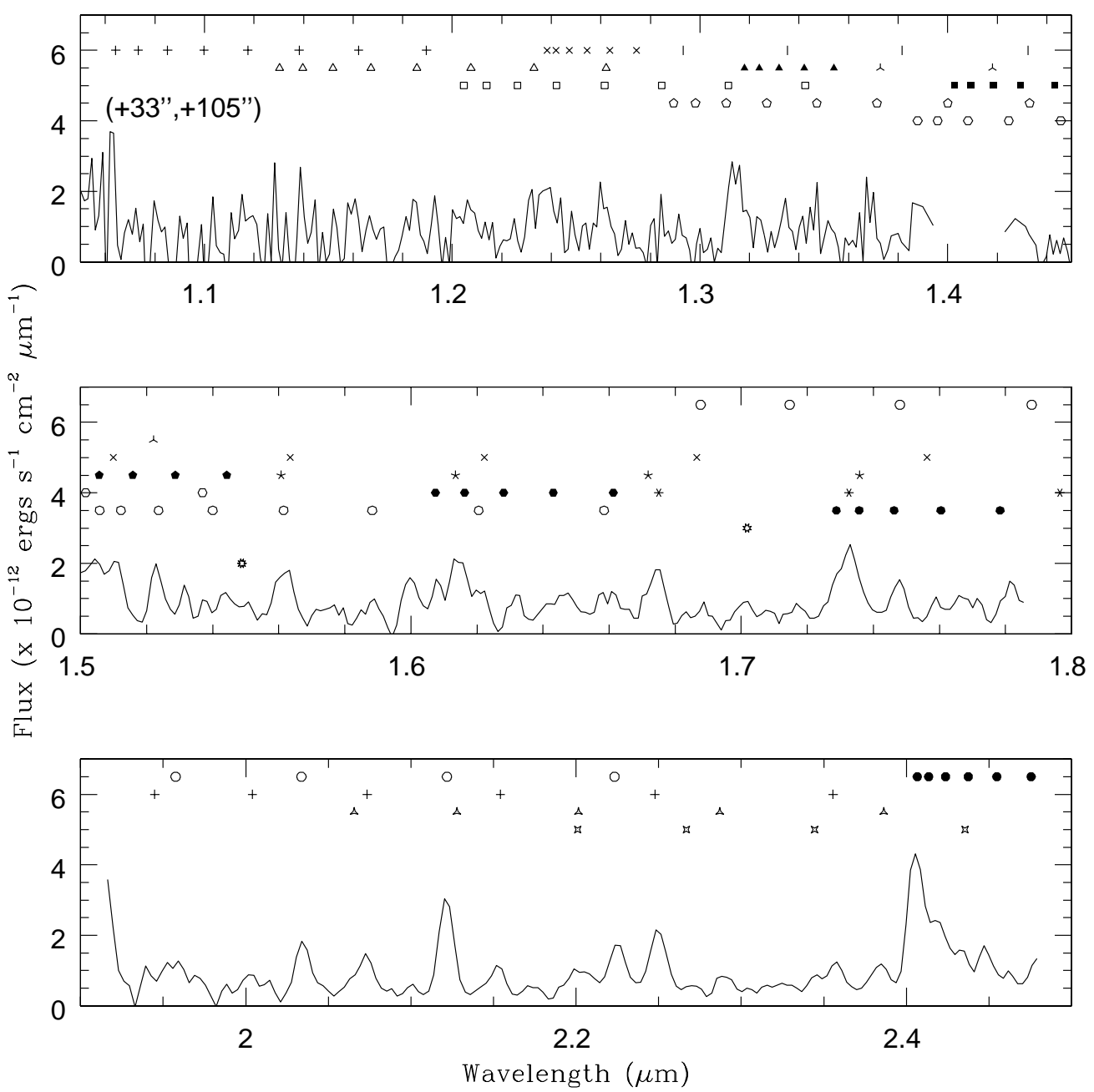

Figure 2-Spectra from $1-2 \cdot 5 \mu \mathrm{m}$ observed at the northern location $\left(+33^{\prime \prime},+105^{\prime \prime}\right)$, in NGC 2023. Symbols are as described for Figure 1.

on the UK Infrared Telescope (UKIRT) on Mauna Kea, Hawaii, using the facility's 7-element grating spectrometer of that time, CGS2. The sky was viewed through a roughly circular field, $5 \cdot 0^{\prime \prime} \times 4 \cdot 5^{\prime \prime}$ in RA and Declination. Observations were obtained by sky-chopping at a few tenths of a hertz on reference positions $120^{\prime \prime}$ from the source.

$\mathrm{J}-(1 \cdot 25 \mu \mathrm{m}), \mathrm{H}-(1 \cdot 65 \mu \mathrm{m})$ and $\mathrm{K}-(2 \cdot 2 \mu \mathrm{m})$ band spectra were obtained at the positions of two bright $\mathrm{H}_{2}$ emission filaments in the north $\left(+33^{\prime \prime},+105^{\prime \prime}\right)$ and south $\left(-11^{\prime \prime},-78^{\prime \prime}\right)$ of the source. Offsets are from HD 37903 at $05^{\mathrm{h}} 39^{\mathrm{m}} 07 \cdot 3^{\mathrm{s}},-02^{\circ} 16^{\prime} 58^{\prime \prime}$ (B1950). The J-band data for $\left(+33^{\prime \prime},+105^{\prime \prime}\right)$ were obtained during the 1991 observations and the other data during the 1989 run. The J- and H-band measurements employed a 633 line $/ \mathrm{mm}$ grating, used in first order for $\mathrm{J}$ and second order for $\mathrm{H}$, and a 300 line $/ \mathrm{mm}$ grating used in second order for $\mathrm{K}$. The spectral resolutions were $0.0041 \mu \mathrm{m}$, $0.0039 \mu \mathrm{m}$ and $0.0098 \mu \mathrm{m}$ for $\mathrm{J}, \mathrm{H}$ and $\mathrm{K}$ bands, respectively.

In the $1 \cdot 38-1 \cdot 43 \mu \mathrm{m}$ and $1 \cdot 80-1 \cdot 94 \mu \mathrm{m}$ wavebands there is severe atmospheric absorption of the radiation. To check the consistency of the measurements on either side of these wavebands, the $\mathrm{J}$ - and $\mathrm{H}$-band spectra were overlapped near $1.5 \mu \mathrm{m}$ and a few bright $\mathrm{H}_{2}$ lines in the $\mathrm{H}$ and $\mathrm{K}$ bands were simultaneously measured with the 633 line $/ \mathrm{mm}$ grating. These flux checks indicated that flux calibrations between bands were consistent with one another to within $20 \%$ and that none of the J-, H- or K-band spectra needed rescaling.

Absolute wavelength calibration and spectral resolution were determined from spectra of an argon lamp. The $\mathrm{H}_{2}$ lines are completely unresolved at our resolution, their FWHM being $<16 \mathrm{~km} \mathrm{~s}^{-1}$ (Burton et al. 1990a). Rest wavelengths for the $\mathrm{H}_{2}$ lines were computed from the $\mathrm{X}^{1} \Sigma_{\mathrm{g}}^{+}$energy level determinations of Dabrowski (1984). We estimate the error in absolute wavelength calibration to be less than one-third of a resolution element.

Absolute fluxes were determined by comparison with observations of the star BS 1543 (spectral type $\mathrm{F} 6 \mathrm{~V}$, apparent magnitudes $J=2 \cdot 35, H=$ $2 \cdot 15$ and $K=2 \cdot 07$ ), obtained adjacent in time to the NGC 2023 observations. We adopted a blackbody temperature of $6100 \mathrm{~K}$ and zero-magnitude 
flux densities of $3 \cdot 0 \times 10^{-7}, 1 \cdot 2 \times 10^{-7}$ and $4 \cdot 0 \times$ $10^{-8} \mathrm{erg} \mathrm{s}^{-1} \mathrm{~cm}^{-2} \mu \mathrm{m}^{-1}$ at $1.25 \mu \mathrm{m}, 1.65 \mu \mathrm{m}$ and $2 \cdot 2 \mu \mathrm{m}$, respectively, to determine the flux density of the calibrator at the wavelength of each line
(Oke \& Schild 1970; Kurucz, Peytremann \& Avrett, 1974). Brackett and Paschen series absorption lines in its spectrum were artificially removed prior to division by it.

Table 1. $\mathrm{H}_{2}$ line identifications and flux densities from spectra

\begin{tabular}{|c|c|c|c|c|c|c|c|}
\hline Line & $\begin{array}{c}\lambda \\
(\mu \mathrm{m})\end{array}$ & $\begin{array}{r}\text { Flux de } \\
\left(10^{-12} \mathrm{erg} \mathrm{s}^{-1}\right. \\
\left(-11^{\prime \prime},-78^{\prime \prime}\right)^{2}\end{array}$ & $\begin{array}{l}\left.\mathrm{cm}^{-2} \mu \mathrm{m}^{-1}\right) \\
\left(+33^{\prime \prime},+105^{\prime \prime}\right)\end{array}$ & Line & $\begin{array}{c}\lambda \\
(\mu \mathrm{m})\end{array}$ & $\begin{array}{r}\text { Flux de } \\
\left(10^{-12} \mathrm{erg} \mathrm{s}^{-1}\right. \\
\left(-11^{\prime \prime},-78^{\prime \prime}\right)^{2}\end{array}$ & $\begin{array}{l}\mathrm{nsity}^{1} \\
\left.\mathrm{~cm}^{-2} \mu \mathrm{m}^{-1}\right) \\
\left(+33^{\prime \prime},+105^{\prime \prime}\right)\end{array}$ \\
\hline $7-4 \mathrm{~S}(1)^{a}$ & $1 \cdot 063070$ & $3 \cdot 1(0 \cdot 7)^{3}$ & $\cdots$ & $6-4 \mathrm{~S}(0)$ & $1 \cdot 536884$ & $1 \cdot 0(0 \cdot 3)$ & $1 \cdot 0(0 \cdot 3)$ \\
\hline $2-0 \mathrm{~S}(7)^{a}$ & $1 \cdot 064143$ & & $\cdots$ & $7-5 \mathrm{~S}(4)$ & $1 \cdot 539990$ & $<0 \cdot 6$ & $1 \cdot 1(0 \cdot 4)$ \\
\hline 2-0 S(4) & $1 \cdot 099817$ & $2 \cdot 3(0 \cdot 6)$ & $<1 \cdot 0$ & $5-3 \mathrm{Q}(6)$ & $1 \cdot 544261$ & $<1 \cdot 0$ & $0 \cdot 7(0 \cdot 3)$ \\
\hline $2-0 \mathrm{~S}(3)$ & $1 \cdot 117492$ & $4 \cdot 0(0 \cdot 6)$ & $2 \cdot 6(0 \cdot 5)$ & $10-7 \mathrm{O}(3)$ & $1 \cdot 548851$ & $<0 \cdot 8$ & $0 \cdot 8(0 \cdot 3)$ \\
\hline $8-5 \mathrm{~S}(3)^{b}$ & $1 \cdot 129215$ & & & $5-3 \mathrm{O}(2)^{m}$ & $1 \cdot 560730$ & $2 \cdot 2(1 \cdot 0)$ & $1 \cdot 6(0 \cdot 8)$ \\
\hline $3-1 \mathrm{~S}(7)^{b}$ & $1 \cdot 130405$ & $2 \cdot 6(0 \cdot 5)$ & $2 \cdot 0(0 \cdot 6)$ & $7-5 \mathrm{~S}(3)^{m}$ & $1 \cdot 561502$ & & \\
\hline $2-0 \mathrm{~S}(2)$ & $1 \cdot 138238$ & $3 \cdot 2(0 \cdot 5)$ & $1 \cdot 7(0 \cdot 3)$ & $5-3 \mathrm{Q}(7)^{m}$ & $1 \cdot 562627$ & & \\
\hline $3-1 \mathrm{~S}(5)^{c}$ & $1 \cdot 151873$ & $4 \cdot 2(0 \cdot 4)$ & $1 \cdot 2(0.5)$ & $4-2 \mathrm{O}(4)^{m}$ & $1 \cdot 563516$ & $2 \cdot 1(1 \cdot 0)$ & $1 \cdot 2(0 \cdot 8)$ \\
\hline $8-5 \mathrm{~S}(1)^{c}$ & $1 \cdot 152436$ & & & $6-4 \mathrm{Q}(1)$ & $1 \cdot 601535$ & $1 \cdot 7(0 \cdot 3)$ & $1 \cdot 5(0 \cdot 3)$ \\
\hline 2-0 S(1) & $1 \cdot 162223$ & $2 \cdot 9(0 \cdot 4)$ & $1 \cdot 8(0 \cdot 3)$ & $6-4 \mathrm{Q}(2)$ & $1 \cdot 607386$ & $1 \cdot 7(0 \cdot 4)$ & $1 \cdot 4(0 \cdot 3)$ \\
\hline $3-1 \mathrm{~S}(4)$ & $1 \cdot 167158$ & $2 \cdot 1(0 \cdot 4)$ & $1 \cdot 2(0 \cdot 3)$ & $5-3 \mathrm{O}(3)$ & $1 \cdot 613535$ & $1 \cdot 6(0 \cdot 4)$ & $1 \cdot 9(0 \cdot 5)$ \\
\hline $3-1 \mathrm{~S}(3)$ & $1 \cdot 185697$ & $3 \cdot 1(0 \cdot 5)$ & $1 \cdot 9(0.5)$ & $6-4 \mathrm{Q}(3)$ & $1 \cdot 616211$ & $2 \cdot 0(0 \cdot 5)$ & $1 \cdot 5(0 \cdot 6)$ \\
\hline $2-0 \mathrm{~S}(0)$ & $1 \cdot 189585$ & $1 \cdot 8(0 \cdot 6)$ & $0 \cdot 4(0 \cdot 4)$ & $7-5 \mathrm{~S}(1)$ & $1 \cdot 620531$ & $0 \cdot 0(0 \cdot 7)$ & $0 \cdot 7(0 \cdot 6)$ \\
\hline${ }^{8-5} \mathrm{Q}(1)^{d}$ & $1 \cdot 201711$ & & & $4-2 \mathrm{O}(5)$ & $1 \cdot 622292$ & $2 \cdot 6(0 \cdot 7)$ & $0.5(0 \cdot 6)$ \\
\hline $4-2 \mathrm{~S}(7)^{d}$ & $1 \cdot 204710$ & & & $6-4 \mathrm{Q}(4)$ & $1 \cdot 628084$ & $<0 \cdot 8$ & $1 \cdot 4(0 \cdot 3)$ \\
\hline $8-5 \mathrm{Q}(2)^{d}$ & $1 \cdot 206846$ & & & $5-3 \mathrm{O}(4)^{n}$ & $1 \cdot 671822$ & $0 \cdot 1(0 \cdot 5)$ & $1 \cdot 0(0 \cdot 5)$ \\
\hline $3-1 \mathrm{~S}(2)^{d}$ & $1 \cdot 207589$ & $2 \cdot 8(0 \cdot 4)$ & $4 \cdot 0(0 \cdot 6)$ & $6-4 \mathrm{O}(2)^{n}$ & $1 \cdot 675020$ & $1 \cdot 7(0 \cdot 3)$ & $1 \cdot 8(0 \cdot 4)$ \\
\hline $4-2 \mathrm{~S}(6)^{e}$ & $1 \cdot 213907$ & & & $6-4 \mathrm{Q}(7)^{o}$ & $1 \cdot 682881$ & $1 \cdot 0(0 \cdot 3)$ & $0 \cdot 6(0 \cdot 3)$ \\
\hline${ }^{8-5} \mathrm{Q}(3)^{e}$ & $1 \cdot 214628$ & $1 \cdot 5(0 \cdot 4)$ & $2 \cdot 1(0 \cdot 5)$ & $4-2 \mathrm{O}(6)^{o}$ & $1 \cdot 686494$ & $0.5(0.8)$ & $0 \cdot 0(0 \cdot 6)$ \\
\hline $4-2 \mathrm{~S}(5)$ & $1 \cdot 226300$ & $2 \cdot 6(0.4)$ & $1 \cdot 1(0 \cdot 3)$ & $11-8 \mathrm{Q}(3)^{\circ}$ & $1 \cdot 687032$ & & \\
\hline $3-1 \mathrm{~S}(1)$ & $1 \cdot 232986$ & $3 \cdot 5(0 \cdot 4)$ & $1 \cdot 8(0 \cdot 2)$ & $1-0 \mathrm{~S}(9)^{o}$ & $1 \cdot 687721$ & $1 \cdot 3(0 \cdot 9)$ & $0.5(0 \cdot 7)$ \\
\hline $2-0 \quad \mathrm{Q}(1)$ & $1 \cdot 238343$ & $2 \cdot 8(0.5)$ & $1 \cdot 9(0 \cdot 3)$ & 8-6 S $(3)^{p}$ & $1 \cdot 701797$ & $1 \cdot 1(0 \cdot 3)$ & $1 \cdot 1(0 \cdot 3)$ \\
\hline $2-0 \mathrm{Q}(2)^{f}$ & $1 \cdot 241937$ & $2 \cdot 4(0 \cdot 5)$ & $1 \cdot 7(0 \cdot 3)$ & $13-9 \mathrm{O}(3)^{p}$ & $1 \cdot 703761$ & & \\
\hline $4-2 \mathrm{~S}(4)^{f}$ & $1 \cdot 242159$ & & & $1-0 \mathrm{~S}(8)$ & $1 \cdot 714660$ & $1 \cdot 0(0 \cdot 3)$ & $<1 \cdot 0$ \\
\hline $2-0 \quad Q(3)$ & $1 \cdot 247324$ & $2 \cdot 2(0 \cdot 4)$ & $0 \cdot 9(0 \cdot 2)$ & $7-5 \mathrm{Q}(1)$ & $1 \cdot 728779$ & $1 \cdot 3(0 \cdot 2)$ & $1 \cdot 3(0 \cdot 4)$ \\
\hline $4-2 \mathrm{~S}(3)^{g}$ & $1 \cdot 261546$ & $5 \cdot 0(0 \cdot 5)$ & $3 \cdot 2(0 \cdot 4)$ & $6-4 \mathrm{O}(3)^{q}$ & $1 \cdot 732637$ & $1 \cdot 7(0 \cdot 4)$ & $2 \cdot 3(0 \cdot 4)$ \\
\hline $3-1 \mathrm{~S}(0)^{g}$ & $1 \cdot 262065$ & & & $7-5 \mathrm{Q}(2)^{q}$ & $1 \cdot 735734$ & & \\
\hline $9-6 \mathrm{~S}(1)^{g}$ & $1 \cdot 262129$ & & & $5-3 \mathrm{O}(5)^{q}$ & $1 \cdot 735888$ & $2 \cdot 4(0 \cdot 3)$ & $1 \cdot 5(0 \cdot 6)$ \\
\hline $2-0 \mathrm{Q}(5)^{g}$ & $1 \cdot 263593$ & & & $7-5 \mathrm{Q}(3)^{r}$ & $1 \cdot 746261$ & & \\
\hline 4-2 S(2) & $1 \cdot 284625$ & $2 \cdot 4(0 \cdot 4)$ & $1 \cdot 1(0 \cdot 2)$ & $1-0 \mathrm{~S}(7)^{r}$ & $1 \cdot 748035$ & $3 \cdot 7(0 \cdot 3)$ & $1 \cdot 3(0 \cdot 2)$ \\
\hline $5-3 \mathrm{~S}(7)$ & $1 \cdot 289366$ & $1 \cdot 7(0 \cdot 4)$ & $0 \cdot 6(0 \cdot 2)$ & $2-1 \mathrm{~S}(5)$ & $1 \cdot 944871$ & $0 \cdot 8(0.5)$ & $0 \cdot 8(0 \cdot 3)$ \\
\hline $2-0 \mathrm{O}(2)$ & $1 \cdot 293229$ & $2 \cdot 2(0 \cdot 4)$ & $1 \cdot 1(0 \cdot 3)$ & $1-0 \mathrm{~S}(3)$ & $1 \cdot 957556$ & $4 \cdot 5(0 \cdot 5)$ & $1 \cdot 7(0 \cdot 4)$ \\
\hline $5-3 \mathrm{~S}(5)^{h}$ & $1 \cdot 310673$ & & & $2-1 \mathrm{~S}(4)$ & $2 \cdot 004072$ & $1 \cdot 8(0 \cdot 4)$ & $0 \cdot 6(0 \cdot 3)$ \\
\hline $4-2 \mathrm{~S}(1)^{h}$ & $1 \cdot 311568$ & $2 \cdot 4(0 \cdot 5)$ & $0 \cdot 7(0 \cdot 4)$ & $1-0 \mathrm{~S}(2)$ & $2 \cdot 033756$ & $3 \cdot 2(0 \cdot 3)$ & $1 \cdot 6(0 \cdot 2)$ \\
\hline $3-1 \mathrm{Q}(1)^{i}$ & $1 \cdot 314102$ & $4 \cdot 0(0.5)$ & $2 \cdot 5(0 \cdot 3)$ & $3-2 \mathrm{~S}(5)^{s}$ & $2 \cdot 065557$ & $1 \cdot 1(0 \cdot 3)$ & $0 \cdot 6(0 \cdot 2)$ \\
\hline $9-6 \mathrm{Q}(1)^{i}$ & $1 \cdot 315821$ & & & $2-1 \mathrm{~S}(3)^{s}$ & $2 \cdot 073510$ & $1 \cdot 7(0 \cdot 3)$ & $0 \cdot 8(0 \cdot 2)$ \\
\hline $3-1 \mathrm{Q}(2)^{i}$ & $1 \cdot 318072$ & $3 \cdot 3(0 \cdot 4)$ & $1 \cdot 7(0 \cdot 2)$ & $1-0 \mathrm{~S}(1)^{t}$ & $2 \cdot 121831$ & $7 \cdot 9(0 \cdot 2)$ & $2 \cdot 9(0 \cdot 2)$ \\
\hline $3-1 \mathrm{Q}(3)^{j}$ & $1 \cdot 324025$ & $3 \cdot 7(0 \cdot 4)$ & $1 \cdot 3(0 \cdot 3)$ & $3-2 \mathrm{~S}(4)^{t}$ & $2 \cdot 127968$ & $0.5(0.4)$ & $0 \cdot 1(0 \cdot 3)$ \\
\hline $5-3 \mathrm{~S}(4)^{j}$ & $1 \cdot 326966$ & & & $2-1 \mathrm{~S}(2)$ & $2 \cdot 154225$ & $1 \cdot 3(0 \cdot 2)^{3}$ & $0 \cdot 7(0 \cdot 1)$ \\
\hline $2-0 \mathrm{O}(3)$ & $1 \cdot 335422$ & $4 \cdot 0(0 \cdot 4)$ & $1 \cdot 7(0 \cdot 2)$ & $3-2 \mathrm{~S}(3)$ & $2 \cdot 201397$ & $1 \cdot 1(0 \cdot 2)$ & $0 \cdot 8(0 \cdot 2)$ \\
\hline $4-2 \mathrm{Q}(7)^{k}$ & $1 \cdot 459198$ & & & $1-0 \mathrm{~S}(0)$ & $2 \cdot 223299$ & $3 \cdot 6(0 \cdot 2)$ & $1 \cdot 5(0 \cdot 1)$ \\
\hline $4-2 \mathrm{O}(2)^{k}$ & $1 \cdot 461130$ & $3 \cdot 0(0 \cdot 4)$ & $1 \cdot 5(0 \cdot 4)$ & $2-1 \mathrm{~S}(1)$ & $2 \cdot 247721$ & $2 \cdot 9(0 \cdot 2)$ & $2 \cdot 0(0 \cdot 1)$ \\
\hline 5-3 Q(1) & $1 \cdot 492938$ & $2 \cdot 3(0 \cdot 3)$ & $1 \cdot 2(0 \cdot 3)$ & $3-2 \mathrm{~S}(2)$ & $2 \cdot 287026$ & $0 \cdot 5(0 \cdot 2)$ & $0 \cdot 4(0 \cdot 2)$ \\
\hline $5-3 \mathrm{Q}(2)$ & $1 \cdot 497988$ & $1 \cdot 5(0 \cdot 4)$ & $0 \cdot 7(0 \cdot 3)$ & 4-3 S(3) & $2 \cdot 344479$ & $0 \cdot 5(0 \cdot 3)$ & $0 \cdot 3(0 \cdot 2)$ \\
\hline 6-4 S(1) & $1 \cdot 501553$ & $2 \cdot 3(0 \cdot 3)$ & $1 \cdot 3(0 \cdot 3)$ & $2-1 \mathrm{~S}(0)$ & $2 \cdot 355629$ & $1 \cdot 2(0 \cdot 3)$ & $0 \cdot 7(0 \cdot 2)$ \\
\hline $5-3 \mathrm{Q}(3)$ & $1 \cdot 505600$ & $2 \cdot 5(0 \cdot 4)$ & $0 \cdot 6(0 \cdot 3)$ & $3-2 \mathrm{~S}(1)$ & $2 \cdot 386447$ & $1 \cdot 3(0 \cdot 3)$ & $0 \cdot 7(0 \cdot 2)$ \\
\hline $4-2 \mathrm{O}(3)$ & $1 \cdot 509865$ & $2 \cdot 8(0 \cdot 2)$ & $2 \cdot 5(0 \cdot 4)$ & $1-0 \mathrm{Q}(1)^{u}$ & $2 \cdot 406594$ & $14 \cdot 4(0 \cdot 5)$ & $3 \cdot 7(0 \cdot 4)$ \\
\hline 5-3 Q(4) & $1 \cdot 515792$ & $1 \cdot 0(0 \cdot 4)$ & $0 \cdot 3(0 \cdot 3)$ & $1-0 \mathrm{Q}(2)^{u}$ & $2 \cdot 413436$ & $3 \cdot 1(0 \cdot 6)$ & $1 \cdot 5(0 \cdot 4)$ \\
\hline $3-1 \mathrm{O}(5)^{l}$ & $1 \cdot 522026$ & $1 \cdot 9(0 \cdot 2)$ & $2 \cdot 2(0 \cdot 3)$ & $1-0 \mathrm{Q}(3)$ & $2 \cdot 423731$ & $5 \cdot 7(0 \cdot 4)$ & $1 \cdot 9(0 \cdot 3)$ \\
\hline $7-5 \mathrm{~S}(5)^{l}$ & $1 \cdot 523598$ & & & $1-0 \quad \mathrm{Q}(4)$ & $2 \cdot 437491$ & $2 \cdot 4(0.5)$ & $1 \cdot 2(0 \cdot 3)$ \\
\hline $5-3 \mathrm{Q}(5)$ & $1 \cdot 528641$ & $1 \cdot 4(0 \cdot 2)^{3}$ & $1 \cdot 0(0 \cdot 3)$ & $1-0 \mathrm{Q}(5)$ & $2 \cdot 454746$ & $2 \cdot 8(0.5)$ & $1 \cdot 1(0 \cdot 4)$ \\
\hline
\end{tabular}

${ }^{1}$ Flux densities have not been corrected for atmospheric transmission (see text).

${ }^{2}$ Positions are RA and Declination offsets from HD 37903, in arcsec.

${ }^{3}$ Values in parentheses are the $1 \sigma$ uncertainties of the line fluxes. Upper limits are $3 \sigma$.

${ }^{a-u}$ Indicates sets of blended lines. Fluxes for blends are the integrated flux of the blend (one flux value tabulated), or are the best-fit multiple Gaussian profiles to the blends (two or more values tabulated). Flux values of the line blends are given opposite the strongest line(s) in the blend according to Black \& van Dishoeck (1987) model 14. 
Table 2. $\mathrm{H}_{2}$ line intensities and column densities from uncontaminated data

\begin{tabular}{|c|c|c|c|c|c|c|}
\hline \multirow[t]{2}{*}{ Line } & \multirow[t]{2}{*}{$\begin{array}{c}\lambda \\
(\mu \mathrm{m})\end{array}$} & \multirow[t]{2}{*}{$\begin{array}{l}E_{\mathrm{u}} \\
(\mathrm{K})\end{array}$} & \multicolumn{2}{|c|}{$\begin{array}{c}\text { Line intensity }{ }^{1} \\
\left(10^{-5} \mathrm{erg} \mathrm{s}^{-1} \mathrm{~cm}^{-2} \mathrm{sr}^{-1}\right)\end{array}$} & \multicolumn{2}{|c|}{$\begin{array}{l}\text { Column density } \\
\left(10^{14} \mathrm{~cm}^{-2}\right)\end{array}$} \\
\hline & & & $\left(-11^{\prime \prime},-78^{\prime \prime}\right)^{2}$ & $\left(+33^{\prime \prime},+105^{\prime \prime}\right)$ & $\left(-11^{\prime \prime},-78^{\prime \prime}\right)$ & $\left(+33^{\prime \prime},+105^{\prime \prime}\right)$ \\
\hline $1-0 \mathrm{Q}(1)$ & $2 \cdot 406594$ & 6149 & $31 \cdot 3(2 \cdot 2)^{3}$ & $8 \cdot 0(1 \cdot 0)$ & $111 \cdot 0(7 \cdot 8)$ & $28 \cdot 5(3 \cdot 6)$ \\
\hline $1-0 \mathrm{Q}(3)$ & $2 \cdot 423731$ & 6951 & $12 \cdot 5(1 \cdot 1)$ & $4 \cdot 2(0 \cdot 8)$ & $68 \cdot 9(6 \cdot 1)$ & $23 \cdot 1(4 \cdot 4)$ \\
\hline $1-0 \mathrm{Q}(4)$ & $2 \cdot 437491$ & 7584 & $4 \cdot 8(1 \cdot 0)$ & $2 \cdot 4(0 \cdot 6)$ & $28 \cdot 1(6 \cdot 0)$ & $13 \cdot 8(3 \cdot 7)$ \\
\hline $1-0 \mathrm{~S}(0)$ & $2 \cdot 223299$ & 6471 & $8 \cdot 2(0 \cdot 7)$ & $3 \cdot 5(0 \cdot 4)$ & $45 \cdot 7(3 \cdot 8)$ & $19 \cdot 5(2 \cdot 1)$ \\
\hline $1-0 \mathrm{~S}(1)$ & $2 \cdot 121831$ & 6951 & $18 \cdot 1(1 \cdot 5)$ & $6 \cdot 6(0 \cdot 7)$ & $70 \cdot 0(5 \cdot 8)$ & $25 \cdot 6(2 \cdot 6)$ \\
\hline $1-0 \mathrm{~S}(2)$ & $2 \cdot 033756$ & 7584 & $7 \cdot 1(0 \cdot 8)$ & $3 \cdot 5(0 \cdot 4)$ & $22 \cdot 6(2 \cdot 6)$ & $11 \cdot 3(1 \cdot 4)$ \\
\hline $1-0 \mathrm{~S}(8)$ & $1 \cdot 714660$ & 14220 & $0 \cdot 9(0 \cdot 3)$ & $<0 \cdot 9$ & $4 \cdot 2(1 \cdot 2)$ & $<4 \cdot 2$ \\
\hline $2-0 \mathrm{O}(3)$ & $1 \cdot 335422$ & 11789 & $3 \cdot 1(0 \cdot 6)$ & $1 \cdot 4(0 \cdot 3)$ & $16 \cdot 2(3 \cdot 0)$ & $7 \cdot 4(1 \cdot 6)$ \\
\hline $2-0 \mathrm{Q}(1)$ & $1 \cdot 238343$ & 11789 & $2 \cdot 7(0 \cdot 7)$ & $1.9(0.5)$ & $11 \cdot 0(2 \cdot 9)$ & $7.5(1.9)$ \\
\hline $2-0 \mathrm{Q}(3)$ & $1 \cdot 247324$ & 12550 & $2 \cdot 2(0 \cdot 6)$ & $0 \cdot 9(0 \cdot 3)$ & $13 \cdot 3(3 \cdot 5)$ & $5 \cdot 6(1 \cdot 8)$ \\
\hline $2-0 \mathrm{~S}(1)$ & $1 \cdot 162223$ & 12550 & $2 \cdot 8(0 \cdot 7)$ & $1 \cdot 7(0 \cdot 5)$ & $10 \cdot 7(2 \cdot 7)$ & $6 \cdot 6(1.9)$ \\
\hline $2-0 \mathrm{~S}(2)$ & $1 \cdot 138238$ & 13150 & $2 \cdot 7(0 \cdot 7)$ & $1 \cdot 6(0.5)$ & $8 \cdot 0(2 \cdot 2)$ & $4 \cdot 8(1 \cdot 4)$ \\
\hline $2-0 \mathrm{~S}(4)$ & $1 \cdot 099817$ & 14764 & $2 \cdot 2(0 \cdot 7)$ & $<1 \cdot 0$ & $4 \cdot 9(1 \cdot 7)$ & $<2 \cdot 2$ \\
\hline $2-1 \mathrm{~S}(1)$ & $2 \cdot 247721$ & 12550 & $6 \cdot 4(0 \cdot 6)$ & $4 \cdot 4(0 \cdot 5)$ & $18 \cdot 1(1 \cdot 7)$ & $12 \cdot 5(1 \cdot 3)$ \\
\hline $2-1 \mathrm{~S}(2)$ & $2 \cdot 154225$ & 13150 & $3 \cdot 0(0 \cdot 5)$ & $1 \cdot 6(0.4)$ & $7 \cdot 3(1 \cdot 1)$ & $3 \cdot 8(0.9)$ \\
\hline $3-1 \mathrm{~S}(1)$ & $1 \cdot 232986$ & 17818 & $3 \cdot 5(0 \cdot 8)$ & $1 \cdot 8(0 \cdot 4)$ & $5 \cdot 7(1 \cdot 3)$ & $2 \cdot 9(0 \cdot 7)$ \\
\hline $3-1 \mathrm{~S}(4)$ & $1 \cdot 167158$ & 19911 & $1.9(0.5)$ & $1 \cdot 2(0 \cdot 4)$ & $2 \cdot 0(0 \cdot 5)$ & $1 \cdot 2(0 \cdot 4)$ \\
\hline $3-1 \mathrm{~S}(7)$ & $1 \cdot 130405$ & 23069 & $2 \cdot 0(0 \cdot 6)$ & $1 \cdot 6(0 \cdot 6)$ & $1 \cdot 9(0 \cdot 6)$ & $1.5(0.6)$ \\
\hline $3-2 \mathrm{~S}(1)$ & $2 \cdot 386447$ & 17818 & $2 \cdot 7(0 \cdot 7)$ & $1 \cdot 6(0 \cdot 5)$ & $8 \cdot 1(2 \cdot 1)$ & $4 \cdot 6(1 \cdot 4)$ \\
\hline $3-2 \mathrm{~S}(2)$ & $2 \cdot 287026$ & 18386 & $1 \cdot 0(0 \cdot 4)$ & $0 \cdot 9(0 \cdot 3)$ & $2 \cdot 6(1 \cdot 0)$ & $2 \cdot 4(0 \cdot 8)$ \\
\hline $4-2 \mathrm{O}(3)$ & $1 \cdot 509865$ & 22079 & $2 \cdot 5(0 \cdot 4)$ & $2 \cdot 2(0 \cdot 5)$ & $3 \cdot 1(0 \cdot 5)$ & $2 \cdot 8(0 \cdot 6)$ \\
\hline 4-2 S(2) & $1 \cdot 284625$ & 23295 & $2 \cdot 4(0 \cdot 6)$ & $1 \cdot 1(0 \cdot 3)$ & $2 \cdot 1(0 \cdot 5)$ & $1 \cdot 0(0 \cdot 3)$ \\
\hline $4-2 \mathrm{~S}(5)$ & $1 \cdot 226300$ & 25623 & $2 \cdot 5(0.6)$ & $1 \cdot 0(0 \cdot 3)$ & $1 \cdot 7(0 \cdot 4)$ & $0 \cdot 7(0 \cdot 2)$ \\
\hline $4-3 \mathrm{~S}(3)$ & $2 \cdot 344479$ & 23955 & $0.9(0.6)$ & $0 \cdot 6(0 \cdot 4)$ & $3 \cdot 0(1 \cdot 8)$ & $1 \cdot 8(1 \cdot 3)$ \\
\hline 5-3 Q(1) & $1 \cdot 492938$ & 26735 & $2 \cdot 0(0 \cdot 4)$ & $1 \cdot 1(0 \cdot 3)$ & $1 \cdot 6(0 \cdot 3)$ & $0 \cdot 9(0 \cdot 2)$ \\
\hline $5-3 \mathrm{Q}(4)$ & $1 \cdot 515792$ & 27878 & $1 \cdot 0(0 \cdot 4)$ & $0 \cdot 3(0 \cdot 2)$ & $1 \cdot 3(0 \cdot 5)$ & $0 \cdot 4(0 \cdot 3)$ \\
\hline $5-3 \mathrm{Q}(5)$ & $1 \cdot 528641$ & 28498 & $1 \cdot 3(0 \cdot 2)$ & $1 \cdot 0(0 \cdot 4)$ & $1 \cdot 8(0 \cdot 3)$ & $1 \cdot 3(0 \cdot 5)$ \\
\hline $5-3 \mathrm{Q}(6)$ & $1 \cdot 544261$ & 29228 & $<1 \cdot 0$ & $0 \cdot 7(0 \cdot 3)$ & $<1 \cdot 3$ & $0.9(0.4)$ \\
\hline $6-4 \mathrm{Q}(1)$ & $1 \cdot 601535$ & 31063 & $1 \cdot 6(0 \cdot 3)$ & $1.3(0.4)$ & $1 \cdot 1(0 \cdot 2)$ & $0.9(0.3)$ \\
\hline $6-4 \mathrm{Q}(2)$ & $1 \cdot 607386$ & 31303 & $1 \cdot 6(0.4)$ & $1 \cdot 2(0 \cdot 3)$ & $1 \cdot 6(0 \cdot 4)$ & $1 \cdot 2(0 \cdot 3)$ \\
\hline $6-4 \mathrm{Q}(4)$ & $1 \cdot 628084$ & 32132 & $<0 \cdot 8$ & $1 \cdot 7(0 \cdot 4)$ & $<1 \cdot 0$ & $2 \cdot 0(0 \cdot 5)$ \\
\hline $6-4 \mathrm{~S}(0)$ & $1 \cdot 536884$ & 31303 & $1 \cdot 0(0 \cdot 3)$ & $1 \cdot 0(0 \cdot 3)$ & $1 \cdot 1(0 \cdot 4)$ & $1 \cdot 1(0 \cdot 4)$ \\
\hline $7-5 \mathrm{~S}(4)$ & 1.539990 & 37220 & $<0 \cdot 6$ & $1 \cdot 0(0 \cdot 4)$ & $<0 \cdot 4$ & $0 \cdot 7(0 \cdot 3)$ \\
\hline 8-6 S(3) & $1 \cdot 701797$ & 40115 & $1 \cdot 0(0 \cdot 3)$ & $1 \cdot 0(0 \cdot 3)$ & $4 \cdot 5(1 \cdot 2)$ & $4.5(1.5)$ \\
\hline $10-7 \mathrm{O}(3)$ & $1 \cdot 548851$ & 44902 & $<0 \cdot 7$ & $0 \cdot 7(0 \cdot 3)$ & $<0 \cdot 6$ & $0 \cdot 7(0 \cdot 3)$ \\
\hline
\end{tabular}

${ }_{1}^{1}$ Intensities have been corrected for atmospheric transmission (see text), but not for extinction.

${ }^{2}$ Positions are RA and Declination offsets from HD 37903, in arcsec.

${ }^{3}$ Values in parentheses are the $1 \sigma$ uncertainties. Upper limits are $3 \sigma$.

\section{Results}

The spectra observed at positions $\left(-11^{\prime \prime},-78^{\prime \prime}\right)$ and $\left(+33^{\prime \prime},+105^{\prime \prime}\right)$ are shown in Figures 1 and 2 respectively. Selected sequences of vibrationalrotational lines are indicated. Table 1 lists all lines that can be identified, together with their flux densities. These were determined by Gaussian fitting the spectra with the FWHM set to the width of the instrumental profile and adjusting the amplitude and wavelength as free parameters, using a least-squares fitting algorithm. In many cases lines were blended, in which case multiple Gaussians were fitted, keeping both line width and separations fixed.

However, the accuracy of this procedure can be severely affected by unequal attenuation of the line source at the specific line frequency compared to the atmospheric attenuation of the continuum source, averaged over a resolution element (see Howe 1992). The atmospheric transmission spectrum from $1-2 \cdot 5 \mu \mathrm{m}$ contains a multitude of unresolved absorption lines, many of which are nearly opaque. Thus, even though the continuum transmission is high, when observed at moderate resolution an individual $\mathrm{H}_{2}$ spectral line coincident in wavelength with a narrow atmospheric absorption line could be attenuated severely, resulting in an underestimate of the $\mathrm{H}_{2}$ line flux after calibration by the standard. Likewise, the strength of an unresolved line may be overestimated if the continuum transmission is low. To reduce such occurrences we modelled the atmospheric transmission across the passbands as observed at Mauna Kea using a model developed by E. Grossman (priv. commun. 1989). The transmission at specific Doppler-shifted wavelengths of $\mathrm{H}_{2}$ line emission from NGC 2023 (which was redshifted by $19 \mathrm{~km} \mathrm{~s}^{-1}$ and $42 \mathrm{~km} \mathrm{~s}^{-1}$ on Nov 28-Dec 1 and Jan 19 , respectively, using a velocity of $+10 \mathrm{~km} \mathrm{~s}^{-1}$ with respect to the local standard of rest for the source) was determined using the model and compared to the mean transmission over the spectral resolution 
of the observation. The reliability of the flux calibration of a particular $\mathrm{H}_{2}$ line could then be estimated from its proximity to a telluric feature. In practice, uncertainties in the widths of the telluric features made the correction of the line flux of an affected $\mathrm{H}_{2}$ line very uncertain, so line fluxes were only determined if the attenuation was less than $10 \%$.

Over 100 lines were observed and identified. However, of these nearly half were too severely blended to allow a reliable determination of individual amplitudes. Of the remainder, 13 were at wavelengths where atmospheric absorption features made the calibration unreliable. Thus roughly one-third of the lines identified could have their specific intensities accurately determined. These are listed in Table 2, converted to erg $\mathrm{s}^{-1} \mathrm{~cm}^{-2} \mathrm{sr}^{-1}$. Also listed are their level column densities, $N_{v, J}$, calculated as

$$
N_{v, J}=\frac{4 \pi \lambda I}{A h c}
$$

where $I$ is the specific intensity of a transition from level $(v, J)$, with radiative decay rate $A$ (taken from Turner, Kirby-Docken \& Dalgarno 1977) emitting a photon of wavelength $\lambda$. Note that no attempt has been made to correct for extinction, although this is likely to be $A_{v} \sim 3-5$ magnitudes (McCartney 1998).

\section{Calibration Error in $8000 \AA$ Data}

We note here, for completeness, that there is a calibration error in the $8000 \AA \mathrm{H}_{2}$ data presented by Burton et al. (1992) for the $\left(-11^{\prime \prime},-78^{\prime \prime}\right)$ position in NGC 2023. The intensities quoted in that paper should be divided by 23 to yield surface brightnesses in $\operatorname{erg~} \mathrm{s}^{-1} \mathrm{~cm}^{-2} \operatorname{arcsec}^{-2}$.

\section{Discussion}

The relatively strong 2-1 S(1) line flux, compared with that of the 1-0 $\mathrm{S}(1)$ line, and the rich spectrum of higher vibrational lines observed clearly indicate that the bulk of the $\mathrm{H}_{2}$ emission arises from fluorescent excitation. This phenomenon was predicted by Black \& Dalgarno (1976) and first observed by Gatley et al. (1987). Our results extend this later work, and provide further detail on the nature of the mechanism.

The 1-0 S(1) line flux is relatively stronger in the southern position, $\left(-11^{\prime \prime},-78^{\prime \prime}\right)$. This is evident in Table 3 , where the intensity of the $\mathrm{H}_{2}$ line with greatest $\mathrm{S} / \mathrm{N}$ in each vibrational level from $v=1$ to $v=6$ is listed. These are relative to that of the 2-1 S(1) line flux at each position. It can be seen that for $v=2-6$ the relative intensities are, within the errors of observation, the same. However, for the $v=1-0 \mathrm{~S}(1)$ line the ratio at $\left(-11^{\prime \prime},-78^{\prime \prime}\right)$ is $2 \cdot 9 \pm 0 \cdot 5$, significantly greater than the value of
Table 3. Representative $\mathbf{H}_{2}$ line ratios for vibrational levels

\begin{tabular}{|c|c|c|c|}
\hline Line & $\left(-11^{\prime \prime},-78^{\prime \prime}\right)^{1}$ & $\left(+33^{\prime \prime},+105^{\prime \prime}\right)^{1}$ & Model $^{1,2}$ \\
\hline $1-0 \mathrm{~S}(1)$ & $2 \cdot 85 \pm 0 \cdot 51$ & $1 \cdot 52 \pm 0 \cdot 31$ & $1 \cdot 60$ \\
\hline $2-1 \mathrm{~S}(1)$ & $1 \cdot 0$ & $1 \cdot 0$ & $1 \cdot 0$ \\
\hline $3-2 \mathrm{~S}(1)$ & $0 \cdot 43 \pm 0 \cdot 15$ & $0 \cdot 36 \pm 0 \cdot 15$ & $0 \cdot 47$ \\
\hline $4-2 \mathrm{O}(3)$ & $0 \cdot 40 \pm 0 \cdot 10$ & $0 \cdot 51 \pm 0 \cdot 16$ & $0 \cdot 67$ \\
\hline $5-3 \mathrm{Q}(1)$ & $0 \cdot 31 \pm 0 \cdot 09$ & $0 \cdot 24 \pm 0 \cdot 09$ & $0 \cdot 68$ \\
\hline $6-4 \mathrm{Q}(1)$ & $0 \cdot 24 \pm 0 \cdot 07$ & $0 \cdot 30 \pm 0 \cdot 12$ & $0 \cdot 53$ \\
\hline
\end{tabular}

${ }^{1}$ Relative intensity compared to the $2-1 \mathrm{~S}(1)$ line. These have not been corrected for differential extinction.

${ }^{2}$ Prediction of Black \& van Dishoeck (1987) model 14.

$1 \cdot 5 \pm 0 \cdot 3$ measured at $\left(+33^{\prime \prime},+105^{\prime \prime}\right)$. The second value is consistent with models for pure fluorescent excitation (Black \& Dalgarno 1976). The first is expected from 'collisional fluorescence' (Sternberg \& Dalgarno 1989; Burton et al. 1990b), whereby collisional de-excitation of fluorescently excited levels increases the $v=0$ and 1 populations over the pure fluorescent value. For this to occur, the gas density has to be above critical $\left(n \gtrsim 10^{5} \mathrm{~cm}^{-3}\right.$ ), with some molecules existing in a hot, self-shielded layer close to the surface of the molecular cloud $\left(A_{v}<1\right)$. Collisional de-excitation of high- $v$ levels can then be significant, as well as thermalisation of the $v=0$ and 1 level populations.

This suggests that the molecular gas in NGC 2023 is clumpy, with some dense gas at the southern position having been heated in a self-shielded layer. We estimate that the far-UV radiation field from HD 37903 , between $6-13 \cdot 6 \mathrm{eV}$, to be $\sim 10^{3}$ times the ambient interstellar value $\left(G_{0}=1 \equiv 1 \cdot 6 \times 10^{-3} \mathrm{erg}\right.$ $\mathrm{s}^{-1} \mathrm{~cm}^{-2}$; Habing 1968) for a projected distance $0 \cdot 18 \mathrm{pc}$ from the star, as at $\left(-11^{\prime \prime},-78^{\prime \prime}\right)$. For $\left(+33^{\prime \prime},+105^{\prime \prime}\right)$ the minimum distance is $0 \cdot 25 \mathrm{pc}$, for which the radiation field would be $\sim 500 G_{0}$. The former radiation field is just sufficient for collisional fluorescence to occur in dense gas; the latter is not, as the gas temperature would not be high enough (Burton et al. 1990b). Thus the data are consistent with a pure fluorescent emission spectrum from the northern position observed, and with some collisional fluorescence at the southern location.

We can examine this conclusion more quantitatively through comparison with predictions of theoretical models, such as that of Burton et al. (1990b). For pure fluorescent emission, $\mathrm{H}_{2}$ line intensities are approximately proportional to the density of the gas and the surface filling factor, $f$, of the emission region in the beam. For the 1-0 $\mathrm{S}(1)$ line it is given to a factor of $\sim 3$ by $I_{1-0 \mathrm{~S}(1)} \sim f n_{4} 10^{-5} \mathrm{erg} \mathrm{s}^{-1} \mathrm{~cm}^{-2} \mathrm{sr}^{-1}$, where $n_{4}$ is the density in units of $10^{4} \mathrm{~cm}^{-3}$. The flux is only weakly dependent on the strength of the far-UV field, the gas being excited over a constant column depth $A_{v} \sim 1$ into the cloud, and an increased pumping rate from a higher far-UV field being balanced by an increased dissociation rate. Thus the observed intensity at $\left(+33^{\prime \prime},+105^{\prime \prime}\right), 7 \times 10^{-5} \mathrm{erg} \mathrm{s}^{-1} \mathrm{~cm}^{-2} \mathrm{sr}^{-1}$, 
is consistent with pure fluorescent emission from molecular gas at a density of a few $\times 10^{4} \mathrm{~cm}^{-3}$, a filling factor of $1-3$ and an extinction at $2 \mu \mathrm{m}$ of $0-1$ magnitudes. At $\left(-11^{\prime \prime},-78^{\prime \prime}\right)$ the radiation field is high enough to heat the surface of the cloud to temperatures of $\sim 1000 \mathrm{~K}$. At the inferred densities, self-shielding of $\mathrm{H}_{2}$ will occur, putting molecular gas close to the surface of the cloud. Over a typical column depth $A_{v} \sim 0 \cdot 1$, there will thus exist hot molecular gas. If, for instance $\left\langle T_{\text {surface }}\right\rangle=750 \mathrm{~K}$, then the intensity of the thermal contribution to the 1-0 S(1) line flux would be $\sim 3 \times 10^{-4} \mathrm{erg} \mathrm{s}^{-1} \mathrm{~cm}^{-2} \mathrm{sr}^{-1}$ from this column, comparable to the $2 \times 10^{-4} \mathrm{erg} \mathrm{s}^{-1} \mathrm{~cm}^{-2} \mathrm{sr}^{-1}$ measured at $\left(-11^{\prime \prime},-78^{\prime \prime}\right)$. However, this temperature is not sufficient to populate the $v=2$ level. The thermal contribution to the $v=2-1 S(1)$ line would be over 1000 times weaker, leaving that of the UV-pump to dominate its line intensity. As concluded above, for $v \geq 2$ fluorescence remains the primary contributor to the line emission.

Also shown in Table 3 are the predictions for the relative $\mathrm{H}_{2}$ line intensities from Black \& van Dishoeck's (1987) model 14, for a fluorescent cascade. We note that, while the observed high-v lines are relatively strong, they are still somewhat weaker than the model predictions for them. In addition, the intensities of higher rotational lines within a given vibrational level tend to be less than those of the lower $J$ lines, contrary to the expectations from Sternberg \& Dalgarno's (1989) model (see also Howe 1992). This indicates that further refinements to the models are still likely to be necessary.

\section{Conclusions}

We have measured over 100 lines of $\mathrm{H}_{2}$ from 1$2.5 \mu \mathrm{m}$ at two positions in the reflection nebula NGC 2023, selecting 35 for which reliable line fluxes and column densities can be inferred. These cover an energy range from 6000-45,000 K above the ground state.

The emission is clearly fluorescent, with the gas in the northern location exhibiting a pure fluorescent spectrum, and that in the southern a collisional fluorescent spectrum. This latter is evidenced by an excess in the $v=1$ population over that for fluorescence, but with pure fluorescent populations for higher levels.

The molecular gas in NGC 2023 forms a clumpy photodissociation region, with the clumps at least as dense as the critical density $\left(\sim 10^{5} \mathrm{~cm}^{-3}\right)$. Those in the southern location experience a sufficiently high far-UV radiation field that the dense gas exists in a hot, self-shielded layer close to the cloud surface $\left(A_{v}<1\right)$. Their excited high $(v \geq 2)$ levels can be collisionally depopulated, while collisions can thermalise the populations in $v=0$ and $v=1$ levels.
The spectrum from $8000 \AA$ to $2.5 \mu \mathrm{m}$ can be readily observed in NGC 2023, and the strengths of several hundred lines of $\mathrm{H}_{2}$ determined. Such a data set could be used for detailed investigations of the nebula and the excitation process. These could include (i) the determination of the ortho-para ratio and its variation with vibrational level, (ii) the derivation of the extinction to the emitting regions by minimising the scatter in a column density-energy level diagram, and (iii) the testing of fluorescent excitation models. Furthermore, the physical parameters it would yield will help to constrain the geometry of NGC 2023. Such an analysis forms the subject of the $\mathrm{PhD}$ dissertation of M. McCartney (1998), which makes use of the data presented here.

\section{Acknowledgments}

We are grateful to the staff of the Joint Astronomy Centre, Hawaii, for their friendly support at the UKIRT telescope where these observations were obtained. MGB wishes to thank the Dublin Institute for Advanced Studies for their hospitality, where much of this paper was written. We appreciate the patience of our colleagues during the long gestation period for this work.

\section{References}

Black, J. H., \& Dalgarno, A. 1976, ApJ, 203, 132

Black, J. H., \& van Dishoeck, E. F. 1987, ApJ, 322, 412

Burton, M. G. 1992, Aust. J. Phys., 45, 463

Burton, M. G., Bulmer, M., Moorhouse, A., Geballe, T. R., \& Brand, P. W. J. L. 1992, MNRAS, 257, 1P

Burton, M. G., Geballe, T. R., Brand, P. W. J. L., \& Moorhouse, A. 1990a, ApJ, 352, 625

Burton, M. G., Hollenbach, D. J., \& Tielens, A. G. G. M. 1990b, ApJ, 365, 620

Burton, M. G., Moorhouse, A., Brand, P. W. J. L., Roche, P. F., \& Geballe, T. R. 1989, in Interstellar Dust: Contributed Papers, IAU Symp. No. 135, NASA CP-3036, 87, ed. L. J. Allamandolla \& A. G. G. M. Tielens

Dabrowski, I. 1984, Can. J. Phys., 62, 1639

DePoy, D. L., Lada, E. A., Gatley, I., \& Probst, R. 1990, ApJ, 356, L55

Draine, B. T., \& Bertoldi, F. 1996, ApJ, 468, 269

Field, D., Gerin, M., Leach, S., Lemaire, J. L., Pineau des Forets, G., Rostas, F., Rouan, D., \& Simons, D. 1994, A\&A, 286, 909

Gatley, I., Hasegawa, T., Suzuki, H., Garden, R., Brand, P., Lightfoot, J., Glencross, W., Okuda, H., \& Nagata, T. 1987, ApJ, 318, L73

Habing, H. J. 1968, Bull. Astron. Inst. Neth., 19, 421

Hasegawa, T., Gatley, I., Garden, R. P., Brand, P. W. J. L., Ohishi, M., Hayashi, M., \& Kaifu, N. 1987, ApJ, 318, L77

Howe, J. E. 1992, PhD Dissertation, University of Texas

Howe, J. E., Jaffe, D. T., Genzel, R., \& Stacey G. J. 1991, ApJ, 373, 158

Jaffe, D. T., Genzel, R., Harris, A. I., Howe, J. E., Stacey, G. J., \& Stutzki, J. 1990, ApJ, 353, 193

Joblin, C., Tielens, A. G. G. M., Allamandolla, L. J., \& Geballe, T. R. 1996, ApJ, 458, 610

Kurucz, R. L., Peytremann, E., \& Avrett, E. H. 1974, Blanketed Model Atmospheres for Early-type Stars (Washington: Smithsonian Institution) 
McCartney, M. 1998, PhD Dissertation, University of Edinburgh

Oke, J. B., \& Schild, R. E. 1970, ApJ, 161, 1015

Steiman-Cameron, T. Y., Haas, M. R., Tielens, A. G. G. M., \& Burton, M. G. 1997, ApJ, 478, 261

Sternberg, A., \& Dalgarno, A. 1989, ApJ, 338, 197
Tielens, A. G. G. M., \& Hollenbach, D. 1985, ApJ, 291, 722 Turner, J., Kirby-Docken, K., \& Dalgarno, A. 1977, ApJS, 35,281

Witt, A. N., \& Malin, D. F. 1989, ApJ, 347, L25

Wyrowski, F., Walmsley, C. M., Natta, A., \& Tielens, A. G. G. M. 1997, A\&A, 324, 1135 